\title{
A frame-shifted gene, which rescued its function by non-natural start codons and its application in constructing synthetic gene circuits
}

Kathakali Sarkar, Sayak Mukhopadhyay, Deepro Bonnerjee, Rajkamal Srivastava and Sangram Bagh* (D)

\begin{abstract}
Background: Frame-shifted genes results in non-functional peptides. Because of this complete loss of function, frame-shifted genes have never been used in constructing synthetic gene circuits.

Results: Here we report that the function of gene circuits is rescued by a frame-shifted gene, which functions by translating from a non-natural start codon. We report a single nucleotide deletion mutation that developed in the $\lambda$-repressor $\mathrm{Cl}$ within a synthetic genetic NOT gate in Escherichia coli during growth and through this mutation, a non-functional synthetic gene circuit became functional. This mutation resulted in a frame-shifted $\mathrm{cl}$, which showed effective functionality among genetic NOT-gates in Escherichia coli with high regulatory ranges (>300) and Hill coefficient (>6.5). The $\mathrm{cl}$ worked over a large range of relative copy numbers between the frame-shifted gene and its target promoter. These properties make this frame-shifted gene an excellent candidate for building synthetic gene circuits. We hypothesized a new operating mechanism and showed evidence that frame-shifted cl was translated from non-natural start codon. We have engineered and tested a series of NOT gates made from a library of $\mathrm{cl}$ genes, each of which starts from a different codon within the first several amino acids of the frame-shifted $\mathrm{cl}$. It is found that one form with start codon ACA, starting from the 3rd codon had similar repression behavior as the whole frame-shifted gene. We demonstrated synthetic genetic NAND and NOR logic-gates with frame-shifted cl. This is the first report of synthetic-gene-circuits made from a frame-shifted gene.
\end{abstract}

Conclusions: This study inspires a new view on frame-shifted gene and may serve as a novel way of building and optimizing synthetic-gene-circuits. This work may also have significance in the understanding of non-directed evolution of synthetic genetic circuits.

Keywords: Frame-shifted gene, Non-natural start codons, Rescued function, Synthetic gene circuits, E. coli, Nondirected evolution of synthetic gene circuit

\section{Background}

The desired behavior of transcriptional synthetic gene circuits is a function of appropriate rate parameters, which are achieved by altering transcription and translational rates, strength of operating sites-transcription factor interaction and their relative copy numbers [1-3]. Transcription and translation rates are changed by altering the sequence of the promoter and ribosome binding

\footnotetext{
*Correspondence: sangram.bagh@saha.ac.in

Biophysics and Structural Genomics Division, Saha Institute of Nuclear Physics, Homi Bhabha National Institute, Block A/F, Sector-I, Bidhannagar, Kolkata 700064, India
}

(c) The Author(s). 2019 Open Access This article is distributed under the terms of the Creative Commons Attribution 4.0 International License (http://creativecommons.org/licenses/by/4.0/), which permits unrestricted use, distribution, and

reproduction in any medium, provided you give appropriate credit to the original author(s) and the source, provide a link to the Creative Commons license, and indicate if changes were made. The Creative Commons Public Domain Dedication waiver (http://creativecommons.org/publicdomain/zero/1.0/) applies to the data made available in this article, unless otherwise stated.

sites (RBS) respectively, during iterative optimization of a gene circuit. The copy numbers are changed by changing the origin of replications in a plasmid $[1,2]$. On the other hand, alteration of operating sites-transcription factor interaction by mutating a transcription factor, through directed evolution [4] is the gold standard approach for optimizing transcriptional gene circuits [5-7]. Directed evolution depends on multiple cycles of generating parallel and random mutations on promoter, RBS or genes, followed by proper screening to identify and isolate the best circuit behaviour. The mutation(s) 
in the gene alters the interaction parameters of the proteins with the other proteins and the DNA. This results change in kinetic parameters of a circuit. Mutations, which bring positive changes in the circuit behaviour are chosen for the next cycle. Mutations, which result negative changes in a circuit or loss of protein functions are no use and automatically discarded during the screening process $[2,4-7]$.

Single nucleotide deletion near the start codon results in a frame-shift, which in turn produces a non-functional peptide. Because of this complete loss of function, frame-shifted genes have never been used in constructing synthetic gene circuits.

Here we report a frame-shift mutated $\lambda$ repressor cI gene, which rescued its function. We hypothesized a new mechanism and showed evidence that the frame-shifted gene might retain its function by getting translated from non-natural start codons. Here we define non-natural start codons as codons, which do not belong to known natural bacterial start codons, including rare ones. We successfully applied this frame-shifted cI in constructing synthetic genetic logic gates, which showed high Hill coefficient, dynamic ranges and workability range. This is also the first report of using a frame-shifted gene in constructing synthetic gene circuits.

\section{Results}

\section{Functional behavior of frame-shifted $\lambda$ repressor $\mathrm{cl}$}

The $\lambda$ repressor $\mathrm{cI}$, in its dimer form works as a strong repressor for the $P_{R}$ promoter [8].During the process of making a genetic NOT gate W1 (Fig. 1a and Table 1), wild type $\mathrm{cI}$ gene was placed under $\mathrm{P}_{\text {LtetO-1 }}$ promoter with a strong RBS [9] and enhanced green fluorescence protein (EGFP) gene was placed under $P_{R}$ promoter with the same RBS, both in a high copy plasmid (pUCori, 500 copies per cell [10]). After ligating the $\mathrm{P}_{\text {LtetO-1 }}-c I$ fragment in the vector containing $\mathrm{P}_{\mathrm{R}}-E G F P$ cassette, $2 \mu \mathrm{l}$ of the ligated product was heat transformed into chemically competent $E$. coli DH5 $\alpha$ Z1 cells, which constitutively expresses lac repressor protein LacI and Tet repressor protein TetR from the bacterial genome [9]. The resultant TetR represses the $\mathrm{P}_{\text {LtetO-1 }}$ promoters present in the plasmids [9]. Therefore, it was expected that in the absence of anhydrotetracycline (aTc), which allosterically binds to TetR proteins and frees the

\section{a}

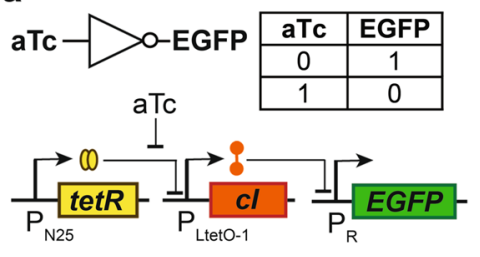

C

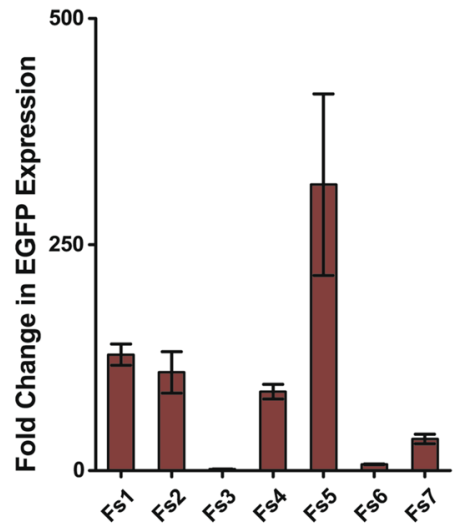

b

wild type $\mathrm{cl}$ : 5'ATGAGCACAAAAAAGAAACC...3'

frame-shifted $c l$ : 5'ATG_GCACAAAAAAGAAACC...3'

d

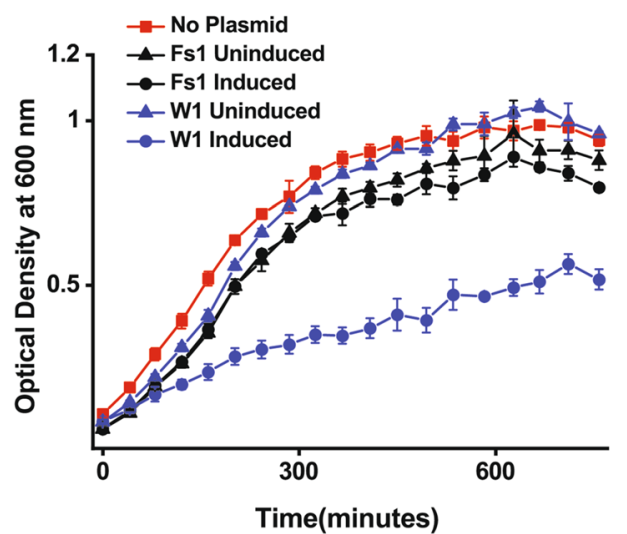

Fig. 1 Frame-shifted $\mathrm{cl}$ and its function. a) Truth table and gene circuit design of the NOT gate. $\mathbf{b}$ ) The partial sequence of wild type and single nucleotide deleted, frame-shifted cl. c) The fold changes in EGFP expression of the NOT gates between output logic level ' 1 ' (high expression), when input is ' 0 ' (absence of aTc) and output logic level 0 (low expression), when input is ' 1 ' ( $200 \mathrm{ng} / \mathrm{ml}$ aTc, saturated concentration, see Fig. 2). The constructs Fs1-7 contain frame-shifted $c l$ gene(Fs1: both frame-shifted $c l$ and $P_{R}$-EGFP in pUC, Fs2: frame-shifted $c l$ in pUC and $P_{R}-E G F P$ in P15A, Fs3: frame-shifted $c l$ in P15A and $P_{R}-E G F P$ in pUC, Fs4: both frame-shifted $c l$ and $P_{R}-E G F P$ in ColE1, Fs5: frame-shifted $c l$ in ColE1 and $P_{R}-E G F P$ in p15A, Fs6: frame-shifted $c l$ in p15A and $P_{R}-E G F P$ in ColE1, Fs7: both frame-shifted $c l$ and $P_{R}-E G F P$ in p15A). d) Comparison of growth curves of

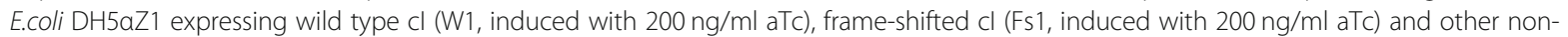
expressing states $(0 \mathrm{ng} / \mathrm{ml})$ that are $\mathrm{W} 1$ uninduced, Fs1 uninduced and without plasmid. W1 contains both wild type $c l$ and $P_{\mathrm{R}}-E G F P$ in $p U C$ 
Table 1 Various NOT gate constructs from wild type (W), frame-shifted (Fs) and truncated versions (T) of $\mathrm{Cl}$ gene (Cl)

\begin{tabular}{|c|c|c|c|}
\hline Constructs & Nature of $\mathrm{Cl}$ & Ori for $\mathrm{P}_{\text {LtetO-1 }}-\mathrm{Cl}$ & Ori for $\mathrm{P}_{\mathrm{R}}-E G F P$ \\
\hline Fs1 & frame-shifted cl & $\mathrm{pUC}$ & $\mathrm{pUC}$ \\
\hline Fs2 & frame-shifted $\mathrm{cl}$ & $\mathrm{pUC}$ & p15A \\
\hline Fs3 & frame-shifted $\mathrm{cl}$ & p15A & $\mathrm{pUC}$ \\
\hline Fs4 & frame-shifted cl & ColE1 & ColE1 \\
\hline Fs5 & frame-shifted cl & ColE1 & p15A \\
\hline Fs6 & frame-shifted cl & p15A & ColE1 \\
\hline Fs7 & frame-shifted cl & p15A & p15A \\
\hline W1 & wild type $\mathrm{cl}$ & pUC & $\mathrm{pUC}$ \\
\hline W2 & wild type $\mathrm{Cl}$ & pUC & p15A \\
\hline W3 & wild type $\mathrm{Cl}$ & p15A & $\mathrm{pUC}$ \\
\hline W4 & wild type $\mathrm{Cl}$ & ColE1 & ColE1 \\
\hline W5 & wild type $\mathrm{Cl}$ & ColE1 & p15A \\
\hline W6 & wild type $\mathrm{cl}$ & p15A & ColE1 \\
\hline W7 & wild type $\mathrm{Cl}$ & p15A & p15A \\
\hline $\mathrm{T} 1$ & Truncated $c\left(T 1^{a} / c l-G\right.$, starting from glycine) & $\mathrm{pUC}$ & p15A \\
\hline $\mathrm{T} 2$ & Truncatedcl(T2 $/ \mathrm{cl}-\mathrm{T} 3$, starting from threonine 3) & pUC & p15A \\
\hline $\mathrm{T} 3$ & Truncated $c /\left(T 3^{\mathrm{a}} / \mathrm{Cl}-\mathrm{K} 4\right.$, starting from lysine 4) & $\mathrm{pUC}$ & p15A \\
\hline $\mathrm{T} 4$ & Truncatedcl(T4 / Cl-K5, starting from lysine 5) & $\mathrm{pUC}$ & p15A \\
\hline T5 & Truncated $c /\left(T 5^{a} / c l-K 6\right.$, starting from lysine 6) & $\mathrm{pUC}$ & p15A \\
\hline T6 & Truncatedcl(T6 $\sigma^{\mathrm{a}} / \mathrm{Cl}$-L8, starting from leucine 8) & $\mathrm{pUC}$ & p15A \\
\hline T7 & Truncatedcl(T7\% / $\mathrm{Cl}-\mathrm{T9}$, starting from threonine 9) & pUC & p15A \\
\hline T8 & Truncated $c\left(T 8^{\mathrm{a}} / \mathrm{cl}\right.$-E11, starting from glutamate 11) & $\mathrm{pUC}$ & p15A \\
\hline T9 & 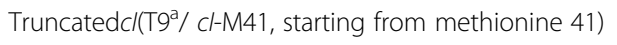 & pUC & p15A \\
\hline
\end{tabular}

${ }^{a}$ The non-natural start codons and their positions are shown in Fig. 3

$\mathrm{P}_{\text {LtetO-1 }}$ promoter for transcription [9], basal level expression of cI protein would be small and could not repress the $\mathrm{P}_{\mathrm{R}}$ promoter. Thus EGFP must be expressed to give rise to green colonies. However, only a small fraction of green fluorescent colonies were observed, with the majority appearing white under blue transillumination.

The plasmids from both fluorescent and non-fluorescent colonies were extracted and sequenced. Results showed a single nucleotide deletion (SND) mutation at the $2^{\text {nd }}$ codon of cI (Fig. 1b) in the plasmids from the green colonies, whereas white colonies showed the correct $\mathrm{CI}$ sequence. This SND resulted in a frame-shift in the $\mathrm{cI}$ gene (Fig. 1b). The deletion found in the cI gene from the green colonies was spontaneous. We did not find mutation in the PCR product of $\mathrm{CI}$ gene before ligation (Additional file 1: Figure S1).

To test the functional ability of this frame-shifted cI, if any, a series of 7 different NOT gate systems (Fs1-Fs7) were constructed, where the absolute as well as relative copy numbers of frame-shifted $\mathrm{cI}$ gene and $P_{R}$ promoter-EGFP gene constructs were varied (Table 1). The constructs followed the general design as in Fig. 1a and were transformed into DH5 $\alpha$ Z1 cells.
Fluorescence from EGFP expression was measured in a multimode reader after growing the diluted cells from overnight culture in fresh Luria-Bertani (LB) medium with appropriate antibiotic for $\sim 16 \mathrm{~h}$ at $37^{\circ}$ C without $(0 \mathrm{ng} / \mathrm{ml})$ and with $(200 \mathrm{ng} / \mathrm{ml})$ aTc, separately (See methods for details).

It was observed that frame-shifted cI effectively represses $P_{R}$ promoter and resulted in reduced EGFP expression with a varying dynamic range changes (from 1.5 times (Fs3) to as high as $>300$ times (Fs5)) between 0 $\mathrm{ng} / \mathrm{ml}$ aTc (input logic level ' 0 ') and $200 \mathrm{ng} / \mathrm{ml}$ (input logic level ' 1 ') aTc concentrations(Fig. 1c and Additional file 1: Figure S2a). Further, E.coli DH5 $\alpha$ Z1 cells expressing frame-shifted cI (Fs1) showed higher growth rate, similar to cells without plasmids in comparison to the cells expressing wild type cI (Fig. 1d). Further, a scatter plot was made to estimate a simple relationship, if any, between the dynamic range and the ratio of approximate average copy numbers of the plasmid carrying frame-shifted $\mathrm{cI}$ and plasmid carrying $\mathrm{P}_{\mathrm{R}}$-EGFP. No simple correlation was found (Additional file 1: Figure S3).

Next, the ultrasensitivity of 5 NOT gates with changes $>30$ times (Fs1, Fs2, Fs4, Fs5 and Fs7) were measured as 
a function of aTc concentration and the experimental behavior was fitted with a Hill function:

$$
[\mathrm{EGFP}]_{\mathrm{ss}}=\mathrm{c}\left(\mathrm{b}+\frac{1}{1+\left(\frac{[\mathrm{aTc}]}{\mathrm{K}}\right)^{\mathrm{n}}}\right)
$$

Where $[E G F P]_{s s}$ represents the normalized fluorescence from EGFP expression, ' $\mathrm{b}$ ' is the basal level fluorescence, $[\mathrm{aTc}]$ is the concentration of aTc in the media, ' $\mathrm{n}$ ' is the Hill coefficient, ' $\mathrm{K}$ ' is the Hill constant and ' $c$ ' is the scaling factor. The Hill coefficient ' $n$ ' is a measure of the ultrasensitivity of a synthetic gene circuit.

Figure 2 shows experimental results along with Hill coefficient (n) values from the fitting. Other parameters are shown in Additional file 1: Table S1. A sharp transition in EGFP expression between low and high aTc concentration with a Hill coefficient (3.6-6.6) was observed. In contrast to the frame-shifted cI constructs (Fs1-Fs7), the similar constructs with wild type cI (W1-W7) were fully repressed under all conditions (Additional file 1: Figure S2b). Even a basal level leakage of wild type cI from fully repressed $\mathrm{P}_{\text {LtetO-1 }}$ promoters (without adding any aTc in the media) in low copy plasmids (p15A ori, approximately 20-30 copies per cell [9] in DH5 $\alpha \mathrm{Z1}$ ) was enough to repress EGFP expression from $P_{R}$ promoters present in high copy plasmids (W3) (pUCori) (Additional file 1: Figure S2b). This experimental observation was also partially supported by thermodynamic calculations using an RBS calculator $[11,12]$, which showed that for the same RBS, the cI gene has $\sim 1170$ times higher translation rate than EGFP (Additional file 1: Table S2). This higher translation rate might produce small but sufficient numbers of cI proteins from the fully repressed $P_{\text {LtetO-1 }}$ promoterto strongly repress the $P_{R}$ promoter.

\section{Hypothesis and evidence of non-natural start codons}

Next, the sequence of the frame-shifted cI was analyzed using a bioinformatics tool (ExPASy Translate, SIB Swiss Institute of Bioinformatics). The first base 'A' in the

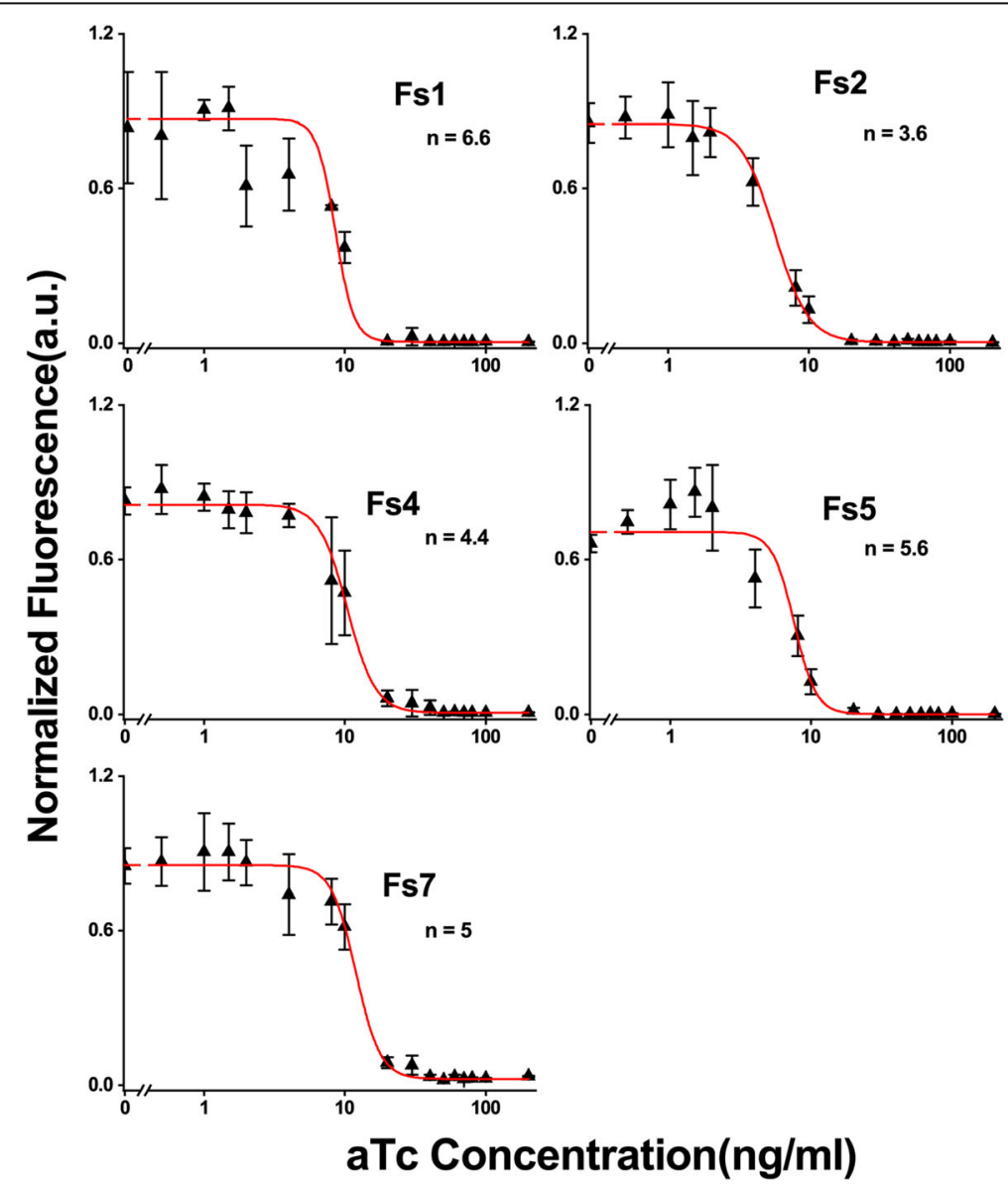

Fig. 2 Ultrasensitivity of NOT gates with frame-shifted cl. Normalized fluorescence from EGFP expression of various NOT gate constructs using frame-shifted $\mathrm{cl}$ as a function of various aTc concentrations. The data were fitted (solid line) with a Hill function (Eq. (1)). The Hill coefficient ' $n$ ' values were shown corresponding to each NOT gate constructs 
second codon of the gene was deleted, resulting in a frame-shift mutation. The resultant protein sequence was completely different from the wild type cI and thus must not have any functional similarity with the wild type cI (Additional file 1: Figure S4a). However, a continuous peptide sequence after 40 amino acids with normal start codon AUG (ATG in gene) was found in frame until the normal stop codon (Additional file 1: Figure S4a). A NOT gate construct (T9) with this truncated version was made and tested. In experiment, this truncated version showed only a 2 -fold reduction in EGFP expression from the $\mathrm{P}_{\mathrm{R}}$ promoter and thus could not explain the high degree of repression (Additional file 1: Figure S4b).

Next, we hypothesized that instead of AUG, the frame-shifted cI might translate from any other codon (non-natural start codons) and bring the protein in frame until the natural stop codon of the wild type cI. We found $8 \mathrm{~N}$-terminus truncated versions of the $\mathrm{cI}$ in frame (Fig. 3a) within first 11 codons for consideration.

To test our hypothesis, a library of plausible (total 8) truncated genes within the first 11 codons with associated non-natural start codons (Fig. 3a) was made (T1-T8 in Table 1). Eight separate constructs were made by replacing frame-shifted cI gene with the truncated genes (T1-T8) in NOT gate construct Fs2 (Table 1, Fig. 3b and Additional file 1: Figure S5d), where frameshifted $\mathrm{cI}$ was under $\mathrm{P}_{\text {LtetO-1 }}$ promoter followed by the RBS and a linker of an optimal length for translational initiation [13, 14]. The new plasmids (T1-T8) were co-transformed with the plasmid carrying $\mathrm{P}_{\mathrm{R}}$-EGFP with p15A Ori same as in Fs2 NOT gate construct (Table 1).

One NOT gate with truncated construct (T2) showed 40-fold repression in EGFP expression from the $P_{R}$

a

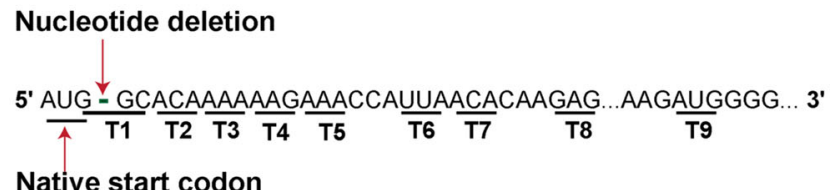

Native start codon

b

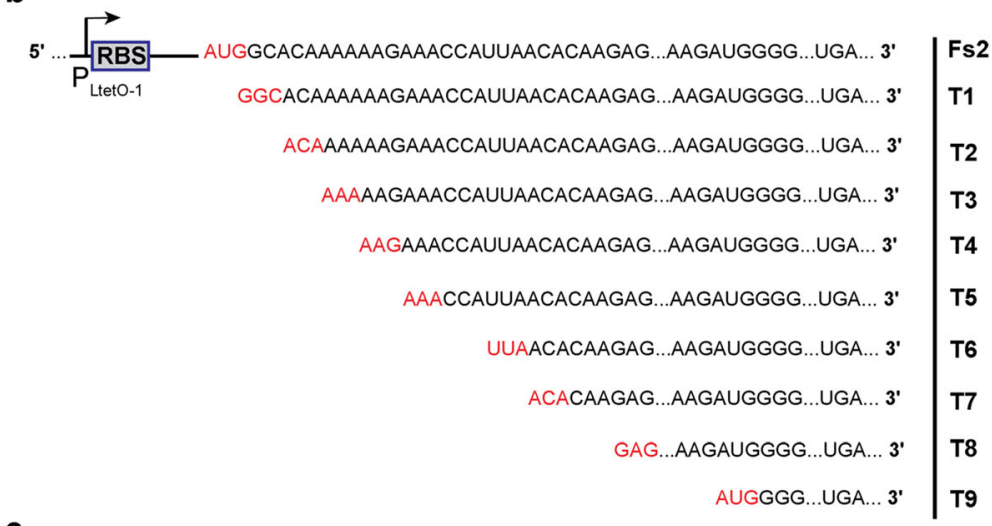

C

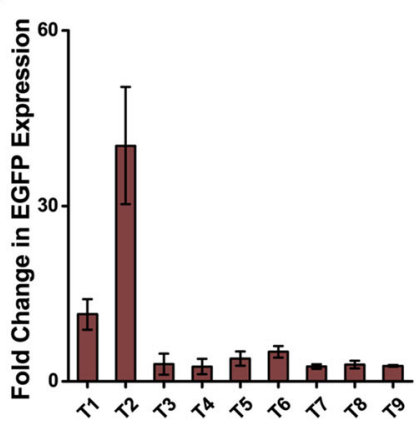

Fig. 3 a) Postulated truncated cl with non-natural start codons (T1-T8). T9 is the AUG start codon after 40th codon. b) Frame-shifted cl in Fs2 NOT gate construct is replaced by individual truncated cls (T1-T9) without changing the $P_{\text {Lteto-1 }}$ promoter, native RBS and the linker region between RBS and ORF, and co-transformed with PR-EGFP carrying plasmid (p15A Ori) to create 9 NOT gates. The start codons of frame-shifted cl and truncated cls are in red and the stop codons (UGA) are also shown. c) The fold change in EGFP expression of the NOT gates with various truncated cls, T1-T9 between output 'ON' (input OFF; $0 \mathrm{ng} / \mathrm{ml}$ aTc) and 'OFF' states (input ON; $200 \mathrm{ng} / \mathrm{ml}$ aTc) 
promoter (Fig. 3c), which matches the repression value ( 34 times) of Fs7.In T2, the truncated cI was translated from 3rd codon ACA and had the first two amino acids (Met and Ser) truncated from the N-terminus. Other truncated constructs with other non-natural start codons (Fig. 3a and Fig. 3b) showed smaller repression ( 211-fold; Fig. 3c and Additional file 1: Figure S6).

\section{Synthetic genetic logic gates with frame-shifted cl}

Next, to demonstrate the functional potential of frame-shifted cI, synthetic genetic universal NOR and NAND logic gates with frame-shifted cI were designed and constructed (Fig. 4). Experimental results showed the NOR logic behavior (Fig. 4b), where only in the absence of both input signals IPTG and aTc (input logic level: 0,0), the output EGFP showed high expression (output logic: 1).

The NAND logic gate was designed as an AND gate followed by a NOT gate. First we made a minimal AND gate by designing, constructing and testing a hybrid synthetic promoter $\mathrm{P}_{\text {LTATAA }}$. This hybrid promoter can be repressed by both LacI and TetR proteins (Fig. 4c) and thus always becomes repressed in DH5 $\alpha \mathrm{Z} 1$ cell and can only be activated in the simultaneous presence of aTc and Isopropyl $\beta$-D-1-thiogalactopyranoside (IPTG), which allosterically binds to LacI and frees the operator sites in the promoter. The design of this hybrid promoter was based on a previous design [15] but used different distances between operator sites, positions and numbers of transcription factor (TF) binding sites (Additional file 1: Table S3). EGFP was placed under this promoter and its successful AND logic behavior was tested (Fig. 4d).

Next, to make a NAND logic system (Fig. 4e), the output gene EGFP of this AND gate was replaced with the frame-shifted $\mathrm{cI}$, placed in a medium copy plasmid (ColE1 ori) and co-transformed with $\mathrm{P}_{\mathrm{R}}-E G F P$ construct (p15A ori) into DH5 $\alpha \mathrm{Z} 1$ cells (Fig. 4e). In this full NAND system, the output of the AND gate (frame-shifted $\mathrm{cI})$ was connected with a NOT gate $\left(\mathrm{P}_{\mathrm{R}}\right.$ promoter) and its output EGFP. Figure $4 \mathrm{f}$ showed the NAND logic behavior, where the output signal is off only when both input signals are present. The difference between OFF and ON states for both NOR and NAND gate were $\sim 40$ times.

\section{Discussion}

The $\lambda$ repressor cI with $P_{R}$ promoter has been used for constructing several gene circuits $[7,16,17]$. However,

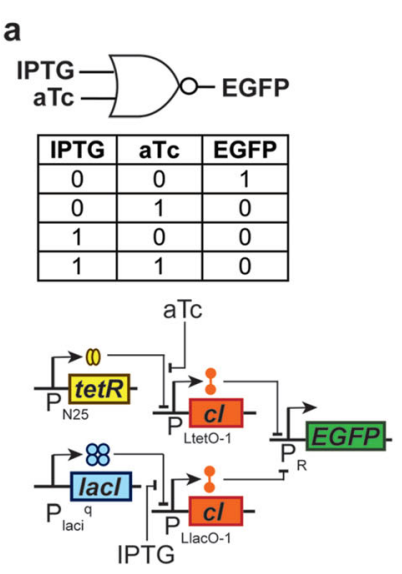

b

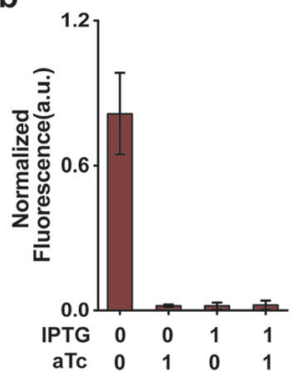

C
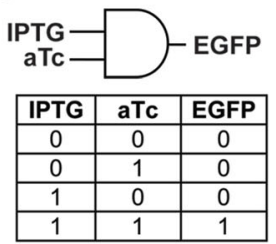

aTc
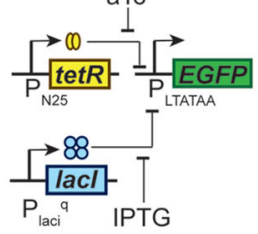

d

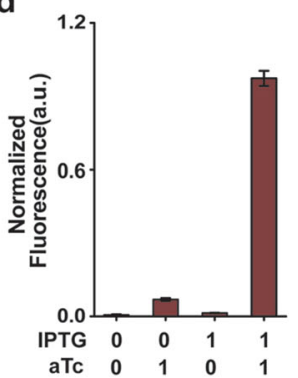

e
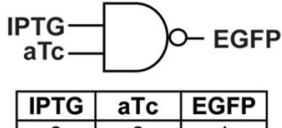

\begin{tabular}{|c|c|c|}
\hline 0 & 0 & 1 \\
\hline 0 & 1 & 1 \\
\hline 1 & 0 & 1 \\
\hline 1 & 1 & 0 \\
\hline
\end{tabular}

aTc
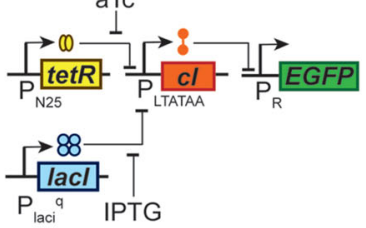

f

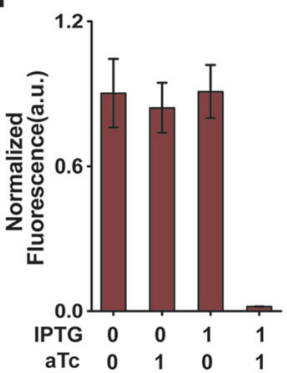

Fig. 4 Logic operations with frame-shifted $\lambda$ repressor. Synthetic NOR and NAND gates are constructed with frame-shifted cl. The truth table and biochemical designs of a) NOR, c) AND and e) NAND logic gates. Experimental behaviour with logic levels for b) NOR, d) AND and $\mathbf{f}$ ) NAND gate is also shown. Each logic gate has two inputs: IPTG and aTc and one output EGFP. The "0" and "1" inputs are basically particular concentrations of both IPTG and aTc (0 mM IPTG for IPTG "0" input, 0 ng/ml aTc for aTc "0" input, 10 mM IPTG for IPTG "1" input and $200 \mathrm{ng} / \mathrm{ml}$ aTc for aTc "1" input) 
the strength of the associated RBS generally needs to be reduced [7]. We showed that even a basal level leakage of wild type cI from fully repressed pLtetO-1 promoter was enough to repress the $P_{R}$ promoter completely. Thus NOT gates made from wild type cI were nonfunctional. However, the function of the NOT gates were rescued when the frame shifted cI was used. The NOT gates constructs created in this study showed high fold change, ultrasensitivity (large Hill coefficient) and normal growth rate as per the best transcriptional NOT gates [18] reported. However, a promoter- repressor pair in a gene circuit may not work effectively in other circuits if there is a mismatch in their relative copy numbers [1]. Similarly, the dynamic range of a specific circuit may not match with the downstream promoter-gene pairs within a larger circuit [1]. In those cases, tedious transcriptional and/or translational optimization is often required to match the [18-21] cellular availability of a repressor genes with respect to the promoters. Here, we have shown that, frame-shifted cI can work over a large range of plasmid copy numbers, as evident from the Fig. 2, which shows successful repression behavior even when the relative numbers of plasmid copy separately carrying frame-shifted $\mathrm{cI}$ and its target promoter are varied in all possible ways (see Table 1). This is one of the most attractive properties of this frame-shifted cI, which most of the other known transcription factor-promoter pairs lack. We think this property of frame-shifted cI originates from the inefficient translation from the non-natural start codons. The inefficient translation would not allow the (truncated) cI protein to reach the saturated level even when we used high copy number plasmid (please see below for more details). Synthetic genetic NOR and NAND gates with frame-shifted cI were successfully demonstrated. The ultrasensitivity observed in those logic circuits is as per the standard transcriptional logic gates reported [22]. It isimportant to note that, in all synthetic gene circuits described here, frame-shifted cI were used with strong promoter and RBS unlike wild type cI found in the literature [7].

This single nucleotide deleted, frame-shifted $\lambda$ repressor cI was produced spontaneously during growth of $E$. coli carrying wild type cI. We hypothesized and showed evidence that frame-shifted cI became functional by producing truncated version of $\mathrm{cI}$ translated from non-natural start codons. It suggests a new mechanism of rescued functionality from a frame-shifted gene. It was previously observed that cI proteins with inserted random linkers in early positions of $\mathrm{N}$-terminal regions showed high to low repression ability against $\mathrm{P}_{\mathrm{L}}$ promoter [23, 24]. However, no studies were performed by replacing or removing AUG (ATG) start codon. The start codons used in our study do not belong to the other lesser used and rare but known start codons in bacteria like GUG, UUG, CUG, AUU, AUC and AUA $[14,25]$. Thus we defined them as non-natural start codons. A recent study [14] showed that translation with varied rate can be achieved with various non natural start codons in super folder green fluorescence protein (sfGFP), which was placed under T7 promoter in E.coli and ACA start codon resulted in a decrease of $\sim 1200$ (in rich defined media) and $\sim 5000$ (in LB media) in sfGFP expression compared to the AUG. Further, the position of this non-natural start codon in frame-shifted CI was 11 nucleotides away from the RBS, which was within the limit for good translation initiation rate in bacteria [13]. This suggested that the frame-shifted cI resulted in functional truncated cI, translating from non-natural ACA codon and its number in the E. coli was reduced proportionally in comparison to wild type cI to give repression with good dynamic range. This was also supported by the observation that we found higher cellular growth rate with frame-shifted cI than wild type cI (Fig. 1d). We reasoned that low amount of truncated but functional cI did not exert high metabolic load, thus it has negligible effect on growth rate.

In our study, we had two interesting observations. First the mutation gave an adaptive advantage as evident from the fact that in induced condition, the plasmids carrying frame-shifted cI had higher growth rate than the plasmid carrying wild type cI. Second, this is an example, where non-directed mutation makes a non-functional gene circuit into a functional one.

\section{Conclusion}

Taken together, our work suggests a new view on a frame-shifted gene in the light of non-natural start codons and inspires future exploration of using frame-shifted genes and non-natural start codons as a new axis for building and optimizing gene expression in synthetic genetic circuits. Further, as this frame-shifted cI requires almost no alteration in its transcription and translation rateswithin a large range of plasmid copy numbers, it could be a better alternative to wild type cI for constructing synthetic gene circuits. This study raises interesting questions about non-directed evolution of synthetic genetic circuits.

\section{Methods}

Promoters, primers, genes, plasmids and bacterial cell strains

Three base plasmids pTA1EGFP, pTA2EGFP and pTC3EGFP were prepared (Additional file 1: Table S4, Additional file 1: Figure S5). EGFP gene, $\mathrm{P}_{\text {LtetO-1 }}$ promoter, ColE1 and p15A origin of replication (ori), ampicillin and chloramphenicol resistance genes were taken from plasmids: pOR-EGFP-12 and pOR-Luc-31 (gift from Prof. David McMillen, University of Toronto, 
Toronto, Canada). The pUCori was PCRed from pmCherry-N1 (Clontech) using Primer_34 and Primer_35 (all the primers used in this study was listed in Additional file 1: Table S5) and incorporated into pTA2EGFP to get pTA1EGFP. All primers were synthesized from Integrated DNA Technologies, Singapore. $P_{R}$ promoter along with RBSwas constructed by self-priming PCR of two primers Primer_9 and Primer_10. EGFP gene was fused to this promoter-RBS fragment by a fusion PCR with Primer_ 8 and Primer_11. This ultimately led to full-length $\mathrm{P}_{\mathrm{R}}$-EGFP construct. This $\mathrm{P}_{\mathrm{R}}$-EGFP construct was flanked by $\mathrm{XhoI}$ and $\mathrm{Xba \textrm {I }}$ and it included the same RBS as that in pTA2EGFP. Hybrid promoter for AND gate, $P_{\text {LtataA }}$ had two TetR binding sites and three LacI binding sites. At first, one DNA fragment containing -10 and -35 hexamers, transcription start site, two TetR binding sites and two LacI binding sites was synthesized by Invitrogen GeneArt Gene Synthesis service, Thermo Fischer. Next we incorporated additional LacI binding site into that synthesized DNA fragment to get full length $\mathrm{P}_{\text {LTATAA }}$ flanked by XhoI and EcoRI sites using Primer_12 and Primer_13. The NAND gate was dependent on this hybrid promoter. Once all the additional promoters were constructed, $\mathrm{P}_{\text {LtetO-1 }}$ was replaced by $\mathrm{P}_{\mathrm{R}}, \mathrm{P}_{\text {LlacO-1 }}$ or $\mathrm{P}_{\text {LTATAA }}$ in the base plasmids as required to generate others.

The $\lambda$ repressor cI gene was PCRed from E. coli K-12 (procured from IMTECH, Chandigarh, India; MTCC number 1302) genomic DNA with appropriate primers (Primer_1-Primer_4) and its truncated variants were PCRed with appropriate primers using wild type cI gene in plasmid as the template. Those were cloned into base plasmids between KpnI and $\mathrm{XbaI}$ under $\mathrm{P}_{\text {LtetO-1 }}, \mathrm{P}_{\text {LlacO-1 }}$ or $\mathrm{P}_{\text {LTATAA }}$ as appropriate (Additional file 1: Figure S5). Frame-shifted cI (cI single nucleotide deletion (SND) mutant) was generated spontaneously during growth. For different constructs, frame-shifted cI was restriction digested and inserted in appropriate plasmidswith varying copy numbers. A DNA fragment containing BamHI and PstI sites was synthesized and placed between AatII and $\mathrm{XhoI}$ in order to introduce two genetic parts within a single plasmid (pLA1SEGFP and PRA1SEGFP)(Additional file 1: Figure S5). We also changed different parts of pLA1SEGFP and PRA1SEGFP to get series of plasmids having various combinations of replication origins (pUC, ColE1 and p15A), antibiotic resistance genes $\left(A p^{\mathrm{r}}\right.$ and $\left.C m^{\mathrm{r}}\right)$, promoters $\left(\mathrm{P}_{\text {LtetO-1 }}, \mathrm{P}_{\text {Llaco-1 }}, \mathrm{P}_{\mathrm{R}}\right.$ and $\left.\mathrm{P}_{\text {LtataA }}\right)$ and genes of interest (EGFP, wild type cI and CI SND mutant) (Additional file 1: Figure S5). We PCRed the second genetic cassette with the primers having PstI and BamHI sites in their flanking regions (Primer_36-Primer_39) for successful incorporation between BamHI and PstI in plasmids. The second genetic cassette incorporated within the DNA fragment was always placed upstream of the first cassette in the opposite direction. All PCR reactions were carried out by either KOD Hot Start DNA polymerase (Merck Millipore), Pfu Turbo Hotstart PCR Master Mix (Agilent Technologies) or Phusion High-Fidelity PCR Master Mix with HF Buffer (New England BioLabs). Plasmids were transformed into chemically competent $E$. coli cells. Cloning and plasmid amplification was performed in DH5 $\alpha$ strain and all experiments were carried out in DH5 $\alpha$ Z1 cells. Transformed cells were grown in LB-Agar, Miller (Difco, Beckton Dickinson) plates with appropriate antibiotics, followed by overnight liquid culture in LB broth from single colonies at $37^{\circ} \mathrm{C}$ with appropriate antibiotics. Plasmids were extracted and sent for sequencing. Plasmids with the correct sequences were used for further experiments. All restriction enzymes and T4 DNA ligase were bought from New England BioLabs; plasmid isolation, gel extraction and PCR purification kits from QIAGEN; ampicillin and chloramphenicol from Himedia; kanamycin, aTc and IPTG from Sigma Aldrich and Abcam. Final concentration of the antibiotics in the growth media was $100 \mu \mathrm{g} / \mathrm{ml}, 34 \mu \mathrm{g} / \mathrm{ml}$ and $50 \mu \mathrm{g} / \mathrm{ml}$ for ampicillin, chloramphenicol and kanamycin respectively. Sequencing of plasmids, genes and promoters were performed by Eurofins Genomics India Pvt. Ltd., Bangalore, India.

\section{Cell growth for genetic constructs characterization}

Cells were grown overnight in LB-liquid media with antibiotics, re-diluted 100 times in fresh LB media with antibiotics and inducers IPTG or aTc as appropriate and again grown for $16 \mathrm{~h}$ at $37^{\circ} \mathrm{C}, \sim 250 \mathrm{rpm}$. Cells were washed twice and re-suspended in phosphate buffered saline (PBS, $\mathrm{pH} 7.4$ ) for taking fluorescence measurements. $0 \mathrm{ng} / \mathrm{ml}$ of aTc (OFF state in input) and $200 \mathrm{ng} /$ $\mathrm{ml}$ aTc (ON state in input) were used for determining $\mathrm{ON}$ and OFF states in fluorescence output. For dose response experiments with aTc, $0 \mathrm{ng} / \mathrm{ml}, 200 \mathrm{ng} / \mathrm{ml}$ and 15 more intermediate aTc concentrations $(0.5 \mathrm{ng} / \mathrm{ml}, 1 \mathrm{ng} /$ $\mathrm{ml}, 1.5 \mathrm{ng} / \mathrm{ml}, 2 \mathrm{ng} / \mathrm{ml}, 4 \mathrm{ng} / \mathrm{ml}, 8 \mathrm{ng} / \mathrm{ml}, 10 \mathrm{ng} / \mathrm{ml}, 20$ $\mathrm{ng} / \mathrm{ml}, 30 \mathrm{ng} / \mathrm{ml}, 40 \mathrm{ng} / \mathrm{ml}, 50 \mathrm{ng} / \mathrm{ml}, 60 \mathrm{ng} / \mathrm{ml}, 70 \mathrm{ng} / \mathrm{ml}$, $80 \mathrm{ng} / \mathrm{ml}$ and $100 \mathrm{ng} / \mathrm{ml}$ ) were used. IPTG were used as $0 \mathrm{mM}$ (for OFF state in input) and $10 \mathrm{mM}$ (for ON state in input) during the characterization of the AND, NOR and NAND gates.

\section{Fluorescence and optical density (OD) measurements}

Cells were diluted in PBS ( $\mathrm{pH}$ 7.4) to reach around $\mathrm{OD}_{600}$ 0.8, loaded onto 96-well multiwell plate (black, Greiner Bio-One) for measurement using Synergy HTX Multi-Mode reader (Biotek Instruments, USA). For fluorescencemeasurements, the cells were excited by a white light source that had been passed through an excitation filter $485 / 20 \mathrm{~nm}$ and emission was collected by 
$516 / 20 \mathrm{~nm}$ bandpass filter with appropriate gain. The $\mathrm{OD}_{600}$ was also measured in the same instrument. The raw fluorescence values were divided by respective $\mathrm{OD}_{600}$ values and thus normalized to the number cells. Auto-fluorescence was measured as average normalized fluorescence of the untransformed DH5 $\alpha \mathrm{Z} 1$ set (no plasmidset) and subtracted from the normalized fluorescence value of the experimental set. Data was been taken for at least 3 biological replicates for each condition.

\section{Data analysis and fitting}

All data analysis and fitting was performed in OriginPro 2015 (OriginLab Corporation, USA). The dose response curves (Fig. 2) were fitted with the Hill equation (Eq. (1)) using built in Levenberg Marquardt algorithm, a damped least squares (DLS) method.

\section{Calculating translational rate in RBS calculator [11, 12]}

Translation initiation rate for EGFP and wild type cI was calculated from "evaluate RBS library" design methods in RBS Library Calculator v2.0, (provided by Prof. Haward Salis, Penn State University, Pennsylvania, USA) considering EcoRI site and its upstream $\mathrm{P}_{\text {LletO-1 }}$ promoter sequence as the pre-sequence, either EGFP or wild type $c I$ as the protein coding sequence, RBS present in base plasmids along with conserved linker GGTACC (KpnI site) as degenerate RBS sequence and E. coli str. K-12 substr. MG1655 as the organism.

\section{Bioinformatics tool}

Analysis of the sequence for wild type cI and cI SND mutant was carried out in ExPASy Translate, SIB Swiss Institute of Bioinformatics.

\section{Additional file}

Additional file 1: Figure S1. Sequencing results for PCRed $\mathrm{cl}$ gene. Figure S2 Characterization of wild type (WT) cl and frame-shifted $\mathrm{cl}$. Figure S3 Correlation between the dynamic range and the approximate ratio of plasmid copy number carrying frame-shifted $\mathrm{Cl}$ and $\mathrm{P}_{\mathrm{R}}$-EGFP construct. Figure S4 Open reading frames of wild type $\mathrm{Cl}$, frame-shifted $\mathrm{Cl}$ and truncated $\mathrm{Cl}$ started from amino acid M41. Figure S5 Generic plasmid maps constructed in this study. Figure S6 Characterization of the NOT gates repression behaviour with postulated truncated variants of Cl. Table S1 Curve fitting parameter values. Table S2 Translation initiation rates for EGFP and $\mathrm{cl}$ calculated from RBS calculator. Table S3 List of promoters. Table S4 List of plasmids. Table S5 List of primers. (DOCX $4535 \mathrm{~kb}$ )

\section{Acknowledgements}

KS, SM, DB and RS acknowledge the support from Saha Institute of Nuclear Physics through PhD scholarships.

\section{Funding}

This work was supported by IBOP Project, Department of Atomic Energy, Govt. of India and Ramanujan Fellowship of SB, Department of Science and Technology, Govt. of India.

\section{Authors' contributions}

This study was conceived by SB. KS and SB designed the studies and the experiments. KS, SM, DB and RS performed the experiments. KS and SB analyzed the data and interpreted the results. SB and KS wrote the manuscript. All authors read and approved the final manuscript.

Ethics approval and consent to participate

Not applicable

\section{Consent for publication}

Not applicable

\section{Competing interests}

The authors declare that they have no competing interests.

\section{Publisher's Note}

Springer Nature remains neutral with regard to jurisdictional claims in published maps and institutional affiliations.

Received: 5 October 2018 Accepted: 20 February 2019

Published online: 01 March 2019

References

1. Brophy JAN, Voigt CA. Principles of genetic circuit design. Nat Methods. 2014;11:508-20.

2. Ang J, Harris E, Hussey BJ, Kil R, McMillen DR. Tuning response curves for synthetic biology. ACS Synth Biol. 2013;2:547-67.

3. Schreiber J, Arter M, Lapique N, Haefliger B, Benenson Y. Model-guided combinatorial optimization of complex synthetic gene networks. MolSyst Biol. 2016;12:899.

4. Arnold FH. Directed evolution: bringing new chemistry to life. Angew Chem Int Ed Engl. 2017;57:2-8.

5. Bassalo MC, Liu R, Gill RT. Directed evolution and synthetic biology applications to microbial systems. Curr Opin Biotechnol. 2016;39:126-33.

6. Ellefson JW, Meyer AJ, Hughes RA, Cannon JR, Brodbelt JS, Ellington AD. Directed evolution of genetic parts and circuits by compartmentalized partnered replication. Nat Biotechnol. 2014;32:97-101.

7. Yokobayashi Y, Weiss R, Arnold FH. Directed evolution of a genetic circuit. Proc Natl Acad Sci U S A. 2002;99:16587-91.

8. Ptashne M. 2004. A genetic switch, Third Edition, Phage Lambda Revisited. Cold Spring Harbor Laboratory Press, U.S.

9. Lutz $\mathrm{R}$, Bujard $\mathrm{H}$. Independent and tight regulation of transcriptional units in Escherichia coli via the LacR/O, the TetR/O and AraC/I1-I2 regulatory elements. Nucleic Acids Res. 1997;25:1203-10.

10. Bagh S, Mazumder M, Velauthapillai T, Sardana V, Dong GQ, Movva AB, et al. Plasmid-borne prokaryotic gene expression: sources of variability and quantitative system characterization. Phys Rev E. 2008;77:021919.

11. Borujeni AE, Channarasappa AS, Salis HM. Translation rate is controlled by coupled trade-offs between site accessibility, selective RNA unfolding and sliding at upstream standby sites. Nucleic Acids Res. 2014;42:2646-59.

12. Salis HM, Mirsky EA, Voigt CA. Automated design of synthetic ribosome binding sites to precisely control protein expression. Nat Biotechnol. 2009; 27:946-50.

13. Chen $H$, Bjerknes M, Kumar R, Jay E. Determination of the optimal aligned spacing between the Shine-Dalgarno sequence and the translation initiation codon of Escherichia coli mRNAs. Nucleic Acids Res. 1994;22:4953-7.

14. Hecht A, Glasgow J, Jaschke PR, Bawazer LA, Munson MS, Cochran JR, et al. Measurements of translation initiation from all 64 codons in E. Coli. Nucleic Acids Res. 2017:45:3615-26.

15. Bagh S, Mandal M, McMillen DR. Minimal genetic device with multiple tunable functions. Phys Rev E. 2010;82:021911.

16. Bagh S, Mandal M, Ang J, McMillen DR. An active intracellular device to prevent lethal disease outcomes in virus infected bacterial cells. BiotechnolBioeng. 2011;108:645-54.

17. Khalil AS, Collins JJ. Synthetic biology: applications come of age. Nat Rev Genet. 2010;11:367-79.

18. Stanton BC, Nielsen AAK, Tamsir A, Clancy K, Peterson T, Voigt CA. Genomic mining of prokaryotic repressors for orthogonal logic gates. Nat Chem Biol. 2014;10:99-105. 
19. Sayut DJ, Niu Y, Sun L. Construction and enhancement of a minimal genetic and logic gate. Appl Environ Microbiol. 2009;75:637-42.

20. Arpino JAJ, Hancock EJ, Anderson J, Barahona M, Stan G-BV Papachristodoulou A, et al. Tuning the dials of synthetic biology. Microbiology. 2013;159:1236-53.

21. Engstrom MD, Pfleger BF. Transcription control engineering and applications in synthetic biology. Synth Syst Biotechnol. 2017;2:176-91.

22. Nielsen AK, Voigt CA. Multi-input CRISPR/Cas genetic circuits that interface host regulatory networks. MolSyst Biol. 2014;10:763.

23. Lieb AM. A fine structure map of spontaneous and induced mutations in the lambda repressor gene, including insertions of IS elements. Mol Gen Genet. 1981;184:364.

24. Rasmussen KK, Frandsen KEH, Erba EB, Pedersen M, Varming AK, Hammer K, et al. Structural and dynamical studies of a truncated variant of $\mathrm{Cl}$ repressor from bacteriophage TP901-1. Sci Rep. 2016:6:29574.

25. Chengguang H, Sabatini P, Brandi L, Giuliodori AM, Pon CL, Gualerzi CO. Ribosomal selection of mRNAs with degenerate initiation triplets. Nucleic Acids Res. 2017:45:7309-25.

Ready to submit your research? Choose BMC and benefit from:

- fast, convenient online submission

- thorough peer review by experienced researchers in your field

- rapid publication on acceptance

- support for research data, including large and complex data types

- gold Open Access which fosters wider collaboration and increased citations

- maximum visibility for your research: over $100 \mathrm{M}$ website views per year

At $\mathrm{BMC}$, research is always in progress.

Learn more biomedcentral.com/submissions 